\title{
Distinct bronchial microbiome precedes clinical diagnosis of lung cancer
}

\author{
Erin A. Marshall ${ }^{1,2}$, Fernando S. L. Filho ${ }^{3}$, Don D. Sin ${ }^{3}$, Stephen Lam ${ }^{1,2}$, Janice M. Leung ${ }^{3^{*}+}$ and Wan L. Lam ${ }^{1,2^{*}+}$ (1)
}

\begin{abstract}
Resident microbial populations have been detected across solid tumors of diverse origins. Sequencing of the airway microbiota represents an opportunity for establishing a novel omics approach to early detection of lung cancer, as well as risk prediction of cancer development. We hypothesize that bacterial shifts in the pre-malignant lung may be detected in non-cancerous airway liquid biopsies collected during bronchoscopy. We analyzed the airway microbiome profile of near 400 patients: epithelial brushing samples from those with lung cancer, those who developed an incident cancer, and those who do not develop cancer after 10-year follow-up. Using linear discriminate analysis, we define and validate a microbial-based classifier that is able to predict incident cancer in patients before diagnosis with no clinical signs of cancer. Our results demonstrate the potential of using lung microbiome profiling as a method for early detection of lung cancer.
\end{abstract}

Keywords: Lung cancer, Early detection, Microbiome, Risk prediction, Liquid biopsy

\section{Background}

Forecasting the $10-15 \%$ of smokers who would develop lung cancer would improve survival rate [1,2]. Smoking weakens the integrity of the bronchial epithelium, rendering the lungs more susceptible to resident microbial changes [3, 4]. Lung tumor and surround non-malignant tissues show differences in microbiome composition, and are both distinguishable from health lungs [4-8]. While lung microbiome profiles are explored as a marker for tumor presence, we see far greater potential in the clinical utility of detecting lung microbiome changes, as an indicator of imminent cancer development, in individuals at risk for cancer before any cancer diagnosis can be made, i.e. prior to clinical detection of tumors [9]. Here, we examined airway microbiomes of 400 individuals

\footnotetext{
*Correspondence: Janice.Leung@hli.ubc.ca; wanlam@bccrc.ca

†Janice M. Leung and Wan L. Lam are co-senior authorship.

${ }^{2}$ Interdisciplinary Oncology Program, University of British Columbia, Vancouver, BC, Canada

${ }^{3}$ Centre for Heart Lung Innovation, St Paul's Hospital, Vancouver, BC, Canada

Full list of author information is available at the end of the article
}

prior to cancer diagnosis, and compared those who do and do not subsequently develop cancer within a $\sim 10$ year follow-up period.

\section{Results \\ Patient cohorts and microbiome profiling}

Bronchscopy-obtained tissue and liquid biopsies (bronchial brushing and washing) are clinically used to monitor high-risk patients. Bronchial brushing samples from bronchoscopy of 352 smokers were retrospectively obtained as part of the Lung Health Study at BC Cancer (BCC) with Research Ethics Board approval [10]. Seven patients had a previous diagnosis of lung cancer that was treated with surgery. Study participants were stratified based on their diagnosis at the time of sampling by bronchoscopy into No Cancer, Incident Cancer and Prevalent Cancer categories for correlative analyses with microbiome attributes (Fig. 1A). Incident Cancer refers to patients who at the time of bronchoscopy did not have cancer, but during follow-up developed incident lung cancer. The 345 samples were randomized and arbitrarily divided into two cohorts such that each category was matched for age, pack-year smoking history,

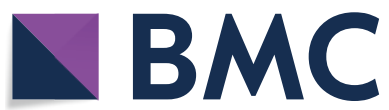

(c) The Author(s) 2022. Open Access This article is licensed under a Creative Commons Attribution 4.0 International License, which permits use, sharing, adaptation, distribution and reproduction in any medium or format, as long as you give appropriate credit to the original author(s) and the source, provide a link to the Creative Commons licence, and indicate if changes were made. The images or other third party material in this article are included in the article's Creative Commons licence, unless indicated otherwise in a credit line to the material. If material is not included in the article's Creative Commons licence and your intended use is not permitted by statutory regulation or exceeds the permitted use, you will need to obtain permission directly from the copyright holder. To view a copy of this licence, visit http://creativecommons.org/licenses/by/4.0/. The Creative Commons Public Domain Dedication waiver (http://creativecommons.org/publicdomain/zero/1.0/) applies to the data made available in this article, unless otherwise stated in a credit line to the data. 
and follow-up time between cohorts: two-thirds used for discovery, and one-third as validation (Cohort $1 n=$ 230, Cohort $2 n=115$; Table 1). These cohorts show no statistically-significant difference in age, pack-year smoking history, lung function, or follow-up time (all $p>0.05$; mean follow-up of 10.2 years).

Airway DNA samples were amplified and profiled at the hypervariable region $\mathrm{V} 4$ of the $16 \mathrm{~S}$ rDNA [11] using a paired-end read chemistry (2x250bp) alongside negative control samples for amplification and sequencing. All samples yielded high-quality sequence data for delineating operational taxonomic units (OTUs, Naïve Bayes clustering) through clustering and alignment to the SILVA reference database (v132) [12, 13]. Reads were processed using the QIIME2 ${ }^{\circledR}$ analysis platform (version 2019.1) [14], and forward reads were trimmed to 231 bases. Organisms identified to be dominant in control samples were excluded from analysis. Microbial community alpha diversity was evaluated (Shannon index, Pielou Evenness, number of OTUs, and Faith's phylogenetic diversity), and no significant difference in diversity between Cohorts 1 and 2, or between groups within each cohort. Further, principal coordinate analysis of Bray Curtis Dissimilarity did not result in separation between participants from Cohorts 1 and 2 (PERMOVA, $p>0.05$; data not shown). Additional samples were obtained from a BC Cancer and St. Paul's Hospital study to form a third cohort for the validation of the microbiome classifier for predicting incident cancers (Cohort $3, n=48$; described in Table 1).

\section{Relating airway microbiome to cancer status}

At the Genus level, relative abundance measures were dominated by Veillonella, Streptococcus, Prevotella, and Paenibacillus in all three categories (182 No Cancer, 36 Incident Cancer and 12 Prevalent Cancer) (Fig. 1B). Taxonomic pattern in Cohort 1 discriminated the Incident Cancer Group from the No Cancer Group (i.e. no lung cancer at any point during follow-up) suggesting that shifts in taxonomic composition related to lung cancer may occur in airways of patients who develop incident lung cancer many months from the date of bronchoscopy. To determine which taxonomic identifiers discriminated patients who developed incident cancers from those who did not develop lung cancer, we used a combination of linear discriminate analysis and effect size modeling (LEfSe; Fig. 1C) [15]. LEfSe was chosen to determine features most likely differentiate groups because in additional to differences in relative abundance, this statistical model captures potential relevance by considering magnitude effect size in the LDA score. Beyond the relative abundance of Bacilli, the features with the strongest association with incident cancer status were concentrated in the Bacilli class, and the relative abundance of Lactobacillales, the Streptococcus genus and associated family, as well as the Paenibacillus genus and associated family.

\section{LMPC classifier predicting incident lung cancer in smokers}

To determine whether the candidate features identified above could be used to build a classifier to predict incident cancer risk, we created a summative score based on the LDA scores and applied them to our cohorts (LMPC; Lung Microbiome Predictor of Cancer). A LDA-weighted combined score was created by multiplying the LDA score value (established in the discovery cohort above) by the relative frequency of the feature in each of the patient sample (Fig. 1D).

In the discovery cohort, the Area Under the Curve of the Receiver Operating Characteristic (AUC) was able to differentiate the Incident Cancer participants from the No Cancer participants $(p<0.0001$, AUC: $0.7057,95 \%$ CI: 0.6118-0.7997; Fig. 1E). Using Cohort 2 as a validation cohort (18 Incident Cancer and 91 No Cancer), this classifier was able to predict cancer incidence. When stratified by the median score, patients with high scores had a significantly higher rates of incident cancer than those with low

\footnotetext{
(See figure on next page.)

Fig. 1 Microbiome-based LMPC classifier identifies cancer onset in independent cohort of patients at risk of lung cancer. A Timeline of bronchial sampling in Cohorts 1-3. B Relative abundance of taxa in the microbial communities Cohorts 1 is shown at genus level of classification. $\mathbf{C}$ Linear discriminant analysis (LDA) scores of taxonomic features included in the LMPC scoring model. A score magnitude of 4 was used as a cut-off for significant features, with a Kruskal-Wallis $p$-value of $<0.05$. Grey bars represent taxa that are higher in relative abundance in No Cancer participants, while blue bars represent taxa with higher relative abundance in Incident Cancer participants. D Formula used to construct LMPC score, where a represents the LDA score value and $x$ represents the relative abundance of each taxa (described in Fig. 1C). E Receiver Operating Characteristic AUC differentiates Incident Cancer participants from No Cancer participants in Cohort 1 ( $p<0.0001$, AUC: 0.7057, 95\% Cl: 0.6118 to 0.7997 ). F When the Incident Cancer $(n=18)$ and No Cancer participants $(n=91)$ in Cohort 2 were combined and stratified by risk score, those with high scores (red) had significantly earlier cancer diagnosis than those with low score (blue). G Within the Incident Cancer patient group, those with high scores demonstrated shorter time to cancer diagnosis than those with low scores. In both analysis, samples were separated into two groups based on the median score value. $\mathbf{H}$ Using a Receiver Operating Characteristic AUC as defined by the score, we are able to differentiate Incident Cancer participants from No Cancer participants in Cohort 2 ( $p=0.0498$, AUC: 0.6503, 95\% Cl: 0.5167-0.7839). I In an independent cohort (Cohort 3), incident cancer participants comprised 4 of the top 5 largest score values. Incident cancer participants are shown in blue, while participants who did not have a diagnosed cancer with follow-up are indicated in black. Cancer onset was assessed using receiver operating curves and log-rank tests, where $p<0.05$ was considered significant
} 
A

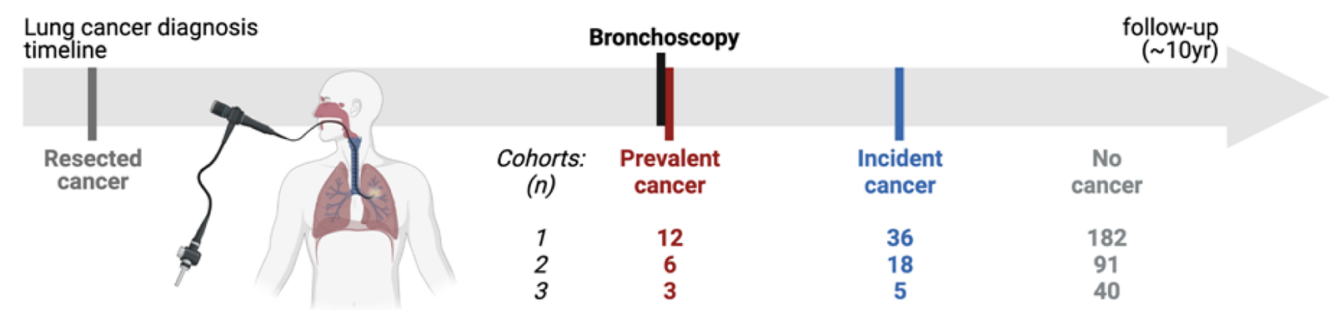

B

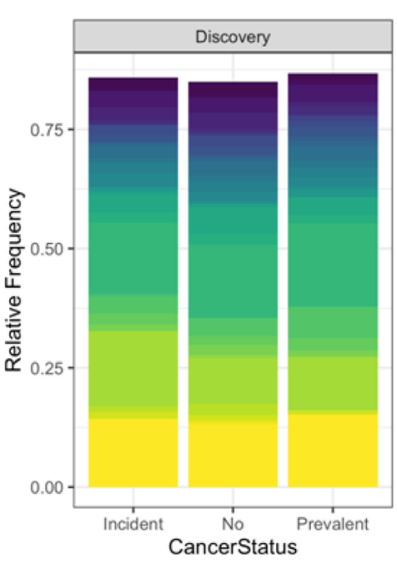

D

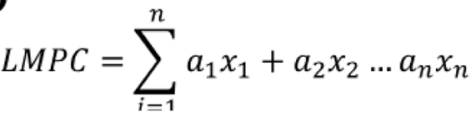

E

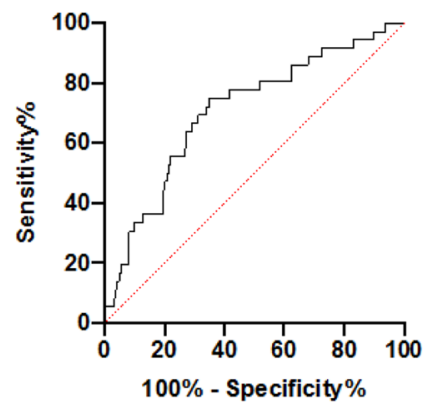

H

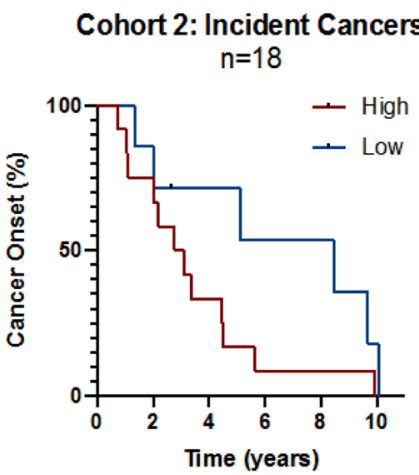

Genus

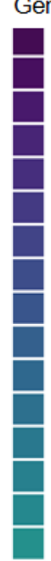

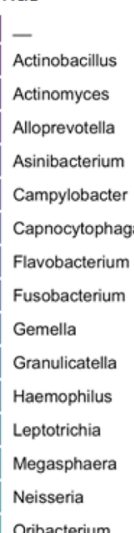

Oribacterium

C

Paenibacillus Porphyromonas Prevotella

Prevotella 6

Prevotella 7 Psychromonas Ralstonia Rothia Selenomonas 3 Staphylococcus Streptococcus

Tropheryma

uncultured

uncultured bacterium Veillonella

F Cohort 2: All asymptomatic $\mathrm{n}=109$
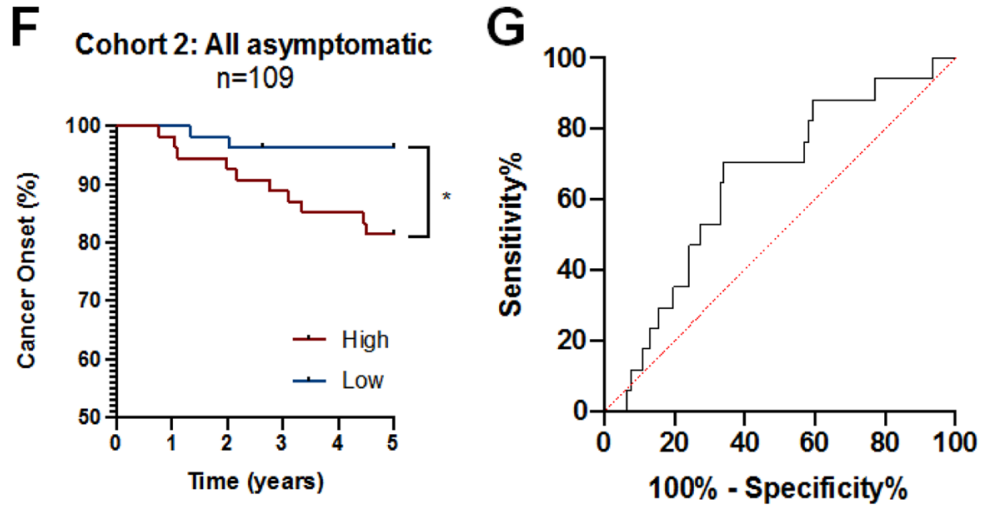

Cohort 3

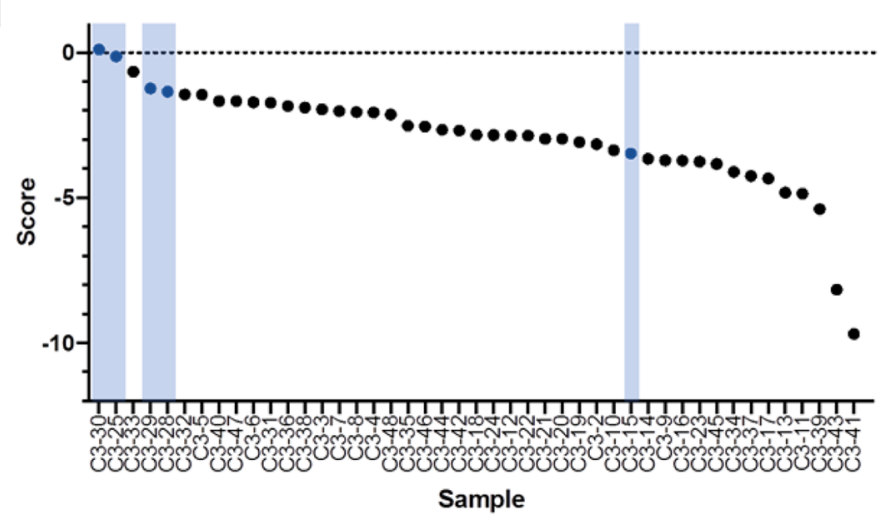

Fig. 1 (See legend on previous page.) 
Table 1 Clinical summary of Cohort $1(n=230), 2(n=115)$, and $3(n=48)$. Cohorts 1 and 2 were sourced from BCC, and clinical characteristics are summarized for incident- $(n=36, n=18)$, prevalent- $(n=12, n=6)$, and no-cancer $(n=182, n=91)$ participants. Cohort 3 was sourced from SPH/BCC and clinical characteristics are summarized for incident- $(n=5)$, prevalent- $(n=3)$, no-cancer $(n=40)$ participants. Patients were randomly assisnged to Cohort 1 or 2 Values are displayed as mean values, unless otherwise specified

\begin{tabular}{|c|c|c|c|c|c|c|c|c|c|}
\hline & \multicolumn{3}{|l|}{ Cohort 1} & \multicolumn{3}{|l|}{ Cohort 2} & \multicolumn{3}{|l|}{ Cohort 3} \\
\hline & Incident & Prevalent & No cancer & Incident & Prevalent & No cancer & Incident & Prevalent & No cancer \\
\hline $\mathbf{N}$ & 36 & 12 & 182 & 18 & 6 & 91 & 5 & 3 & 40 \\
\hline Age (mean) & 64.4 & 62.1 & 62.1 & 64.6 & 65.5 & 61.8 & 64.6 & 64.3 & 62.6 \\
\hline Sex (\% female) & 44.4 & 50 & 46.2 & 50 & 83.3 & 45.1 & 40 & 66.6 & 47.5 \\
\hline \multicolumn{10}{|l|}{ Smoking status (mean \%) } \\
\hline Current & 50 & 25 & 45.6 & 55.6 & 33.3 & 47.3 & 20 & 33.3 & 47.5 \\
\hline Ex & 50 & 75 & 54.4 & 44.4 & 66.7 & 52.7 & 60 & 0.0 & 52.5 \\
\hline Never & 0 & 0 & 0 & 0 & 0 & 0 & 20 & 33.3 & 0 \\
\hline Pack-years smoked (mean) & 51.1 & 48 & 47 & 55.3 & 47 & 44.8 & 50.7 & 37.8 & 48 \\
\hline \multicolumn{10}{|l|}{ Lung function (mean) } \\
\hline FEV1/FVC & 67.3 & 62 & 71.3 & 66 & 65.2 & 72.1 & 73.6 & 79 & 67 \\
\hline FEV1 & 2.5 & 2.1 & 2.6 & 2.3 & 1.8 & 2.6 & 2.3 & 2.1 & 2.4 \\
\hline FEV1 $\%$ predicted & 78.5 & 71.4 & 84.3 & 85.2 & 68.6 & 85.2 & 88 & 83.3 & 77.6 \\
\hline \multicolumn{10}{|l|}{ COPD status (mean \%) } \\
\hline No COPD & 36.1 & 33.3 & 57.1 & 38.9 & 33.3 & 63.7 & 80 & 100 & 45 \\
\hline Mild & 19.4 & 8.3 & 18.1 & 16.7 & 0 & 17.6 & 0 & 0 & 5 \\
\hline Moderate & 38.9 & 25 & 20.9 & 33.3 & 33.3 & 16.5 & 20 & 0 & 35 \\
\hline Severe & 2.8 & 16.7 & 2.7 & 11.1 & 16.7 & 1.1 & 0 & 0 & 15 \\
\hline Very Severe & 0 & 0 & 0 & 0 & 0 & 0 & 0 & 0 & 0 \\
\hline \multicolumn{10}{|l|}{ Follow-up time (mean, yr) } \\
\hline Total & 9.7 & 9.5 & 10 & 10.1 & 8.4 & 10.1 & 7.6 & 7 & 9.8 \\
\hline To cancer & 4.2 & N/A & N/A & 4.3 & N/A & $\mathrm{N} / \mathrm{A}$ & 1.9 & N/A & N/A \\
\hline \multicolumn{10}{|l|}{ Cancer type } \\
\hline LUAD & 42.4 & 88.9 & & 38.9 & 66.7 & & 100 & 100 & \\
\hline LUSC & 12.1 & 11.1 & & 16.7 & 33.3 & & 0 & 0 & \\
\hline NSCLC & 9.1 & 0 & & 11.1 & 0 & & 0 & 0 & \\
\hline SCLC & 12.1 & 0 & & 5.6 & 0 & & 0 & 0 & \\
\hline Other & 24.2 & 0 & & 27.8 & 0 & & NA & NA & \\
\hline
\end{tabular}

scores $(p=0.01)$ (Fig. 1F). Further, when clinical features are incorporated to adjust for participant age, smoking history (pack-years smoked), and lung function (FEV1\% predicted), the LMPC score can identify patients who will develop lung cancer (Cox proportional hazards model; log-rank test, $p=0.004)$. Again, the AUC was able to differentiate the Incident Cancer from No Cancer participants $(p=0.0498$, AUC: 0.6503, 95\% CI: 0.5167-0.7839) (Fig. 1G). Further, within the Incident Cancer subset of Cohort 2, patients with higher scores had a trend towards faster cancer incidence than those with low scores (log-rank test, $p=0.05$; adjusted for clinical co-variants as above) (Fig. 1H).

\section{Application of LMPC classifier to an independent cohort} A third cohort was processed and sequenced independently from Cohorts 1 and 2 (40 No Cancer, 5 Incident
Cancer). After applying the scoring system to Cohort 3, 4/5 Incident Cancer participants had the higher risk scores, indicating that these individuals may be at the highest risk of developing cancer (Fig. 1I). Indeed, they developed lung cancer on average 16 months from the time of sampling, while the case with low score did not developed cancer until 53 months. The AUC readily differentiated Incident Cancer from No Cancer participants ( $p=0.0103$, AUC: $0.8550,95 \%$ CI: 0.6180-1.000).

\section{Discussion}

Distinguishing the 10-15\% of smokers who would develop lung cancer from those who will not presents a clinical challenge. While the consensus of specific taxonomic classifiers associated with lung cancer remains contentious, it is clear that the microbiome is involved in human biological processes, and plays a functional role 
in cancer pathogenesis. However, the timing of these microbial community alterations with respect to cancer onset remains unknown and has tremendous clinical implications in advancing early detection of lung cancer. Here, we showed that there are significant detectable changes in the lung microbiome in participants who, at the time of bronchoscopy, did not have cancer but who subsequently developed lung cancer during follow-up (i.e. the Incident Cancer patients). These data thus propose a microbial-DNA-based classifier to predict incident cancer cases in our cohort of current and former smokers.

We identified taxonomic features (changes in Cohort 1) that yielded the largest LDA score and developed a classifier, which was able to distinguish cancer status. The classifier model was validated in Cohort 2 , demonstrating its ability to distinguish Incident Cancer from No Cancer and thus enabling prediction of incident cancer in smokers who are otherwise asymptomatic (Fig. 1D). When stratified by the LMPC classifier, participants with a high score have a significantly shorter time to cancer onset than those with a low score in a combined group of No-Cancer and Incident Cancer participants. Of note, the absolute risk in this cohort is consistent with cancer onset rates in "high-risk" individuals, suggesting that these findings may be applicable to a broader at-risk community. Within the Incident Cancer group (in Cohort 2) those samples with high scores (separated by median) had earlier cancer onset than those with low scores (Fig. 1F).

When this same scoring was applied to a third cohort that was sequenced independently, 4/5 Incident Cancer participants were identified to have the highest LMPC scores and developed cancer in a mean time of 1.29 years from the time of sampling (Fig. 1H). Upon further examination, the fifth Incident Cancer sample (C3-15) with the lower score was found to have a much longer timeto-cancer diagnosis of 4.43 years following the airway sample collection. Since this proposed classifier is able to distinguish participants who will develop cancer over time from those who will not, it is reasonable to expect that this score is more predictive the closer the sample procurement is to cancer diagnosis. The behavior of this patient is consistent with this expectation, indicating that this risk score model may be most applicable closer to cancer onset.

Our results demonstrate that specific changes in airway liquid biopsy (brushings) samples, obtained during clinical examination by bronchoscopy, in fact, occur before clinical cancer diagnosis. Lung microbiome shifts can serve as a marker in advance of the clinical phenotypes detectable by low-dose CT scans. We have defined a microbiome-based classifier that can predict incident cancer with follow-up from patients having no clinical signs of cancer. As lung-resident microbiomes are associated with smoking status, and this study describes cohorts of ever-smokers and former smokers, future work should examine if this signature can be applied to assess cancer onset in individuals without a history of smoking history. Our findings provide strong rationale for developing microbiome-based liquid biopsy technology to prioritize at-risk individuals for clinical attention.

\section{Abbreviations \\ AUC: Area under the curve; BCC: BC Cancer; LDA: Linear discriminant analysis; LDCT: Low-dose computed tomography; LEfSe: Linear discriminant analysis effect size; LHS: Lung health study; LUAD: Lung adenocarcinoma; LUSC: Lung squamous cell carcinoma; FEV: Forced expiratory volume; FEV1: Forced expiratory volume in 1 second; FVC: Forced Vital Capacity; NM: Non-malignant; NSCLC : Non-small cell lung cancer; OTU: Operational taxonomic unit; SCLC: Small cell lung cancer; SPH: St Paul's Hospital.}

\section{Acknowledgments}

We thank members of the Lam lab who provided critical insight and feedback throughout the duration of the work. The authors would especially like to thank Julia Yang for her assistance in sample processing.

\section{Authors' contributions}

EAM, DDS, JML and WLL developed the conceptual design of the article. EAM, FSLF, SL, JML and WLL established methodologies. EAM, FSLF and SL investigated sample cohorts. EAM was responsible for data generation and analysis, and produced the written and graphical content. All authors contributed to data interpretation. All authors discussed, read, edited, and approved the final version of the manuscript.

\section{Funding}

The presented work was supported by funds from the Canadian Institutes for Health Research (grant FDN-143345) and the BC Cancer Foundation. EAM was supported by a Vanier Canada Graduate Scholarship.

\section{Availability of data and materials}

All sequencing data generated in this study have been deposited in the Sequence Read Archive (SRA) of the National Center for Biotechnology Information (NCBI).

\section{Declarations}

\section{Ethics approval and consent to participate}

Samples were collected under written and informed participant consent, and according to approved ethical guidelines from the University of British Columbia-BC Cancer Research Ethics Board (H17-01846, H08-01132, H0261177, H07-01393 and H06-00049).

\section{Consent for publication}

Not applicable.

\section{Competing interests \\ Authors declare that they have no competing interests.}

\section{Author details}

${ }^{1}$ Department of Integrative Oncology, BC Cancer Research Institute, Vancouver, BC, Canada. ${ }^{2}$ Interdisciplinary Oncology Program, University of British Columbia, Vancouver, BC, Canada. ${ }^{3}$ Centre for Heart Lung Innovation, St Paul's Hospital, Vancouver, BC, Canada.

Received: 19 December 2021 Accepted: 21 February 2022

Published online: 07 March 2022 


\section{References}

1. Herbst RS, Morgensztern D, Boshoff C. The biology and management of non-small cell lung cancer. Nature. 2018;553(7689):446-54.

2. Bruder C, Bulliard JL, Germann S, Konzelmann I, Bochud M, Leyvraz M, et al. Estimating lifetime and 10-year risk of lung cancer. Prev Med Rep. 2018;11:125-30.

3. Hou W, Hu S, Li C, Ma H, Wang Q, Meng G, et al. Cigarette smoke induced lung barrier dysfunction, EMT, and tissue remodeling: a possible link between COPD and lung cancer. Biomed Res Int. 2019:2019:2025636.

4. Wong LM, Shende N, Li WT, Castaneda G, Apostol L, Chang EY, et al. Comparative analysis of age- and gender-associated microbiome in lung adenocarcinoma and lung squamous cell carcinoma. Cancers (Basel). 2020;12(6):1447

5. Riquelme E, Zhang Y, Zhang L, Montiel M, Zoltan M, Dong W, et al. Tumor microbiome diversity and composition influence pancreatic cancer outcomes. Cell. 2019;178(4):795-806 e12.

6. Greathouse KL, White JR, Vargas AJ, Bliskovsky WV, Beck JA, von Muhlinen $\mathrm{N}$, et al. Interaction between the microbiome and TP53 in human lung cancer. Genome Biol. 2018;19(1):123.

7. Jin C, Lagoudas GK, Zhao C, Bullman S, Bhutkar A, Hu B, et al. Commensal microbiota promote lung cancer development via $\gamma \delta T$ cells. Cell. 2019;176(5):998-1013.e16.

8. Poore GD, Kopylova E, Zhu Q, Carpenter C, Fraraccio S, Wandro S, et al Microbiome analyses of blood and tissues suggest cancer diagnostic approach. Nature. 2020;579(7800):567-74

9. Black WC, Gareen IF, Soneji SS, Sicks JD, Keeler EB, Aberle DR, et al. Costeffectiveness of CT screening in the national lung screening trial. N Engl J Med. 2014;371(19):1793-802.

10. Tammemagi MC, Lam SC, McWilliams AM, Sin DD. Incremental value of pulmonary function and sputum DNA image cytometry in lung cancer risk prediction. Cancer Prev Res. 2011:4(4):552-61.

11. Kozich JJ, Westcott SL, Baxter NT, Highlander SK, Schloss PD. Development of a dual-index sequencing strategy and curation pipeline for analyzing amplicon sequence data on the MiSeq Illumina sequencing platform. Appl Environ Microbiol. 2013;79(17):5112-20.

12. Rognes T, Flouri T, Nichols B, Quince C, Mahé F. VSEARCH: a versatile open source tool for metagenomics. PeerJ. 2016;4:e2584

13. Quast C, Pruesse E, Yilmaz P, Gerken J, Schweer T, Yarza P, et al. The SILVA ribosomal RNA gene database project: improved data processing and web-based tools. Nucleic Acids Res. 2013;41(Database issue):D590-6.

14. Bolyen E, Rideout JR, Dillon MR, Bokulich NA, Abnet CC, Al-Ghalith GA, et al. Reproducible, interactive, scalable and extensible microbiome data science using QIIME 2. Nat Biotechnol. 2019;37(8):852-7.

15. Segata N, Izard J, Waldron L, Gevers D, Miropolsky L, Garrett WS, et al. Metagenomic biomarker discovery and explanation. Genome Biol. 2011;12(6):R60

\section{Publisher's Note}

Springer Nature remains neutral with regard to jurisdictional claims in published maps and institutional affiliations.

Ready to submit your research? Choose BMC and benefit from:

- fast, convenient online submission

- thorough peer review by experienced researchers in your field

- rapid publication on acceptance

- support for research data, including large and complex data types

- gold Open Access which fosters wider collaboration and increased citations

- maximum visibility for your research: over $100 \mathrm{M}$ website views per year

At BMC, research is always in progress.

Learn more biomedcentral.com/submissions 